\title{
Bayesian source identification using local priors
}

\author{
M. Aucejo ${ }^{\mathrm{a}}$, O. De Smet ${ }^{\mathrm{a}}$ \\ ${ }^{a}$ Structural Mechanics and Coupled Systems Laboratory, Conservatoire National des Arts \\ et Métiers, 2 Rue Conté, 75003 Paris, France
}

\begin{abstract}
This paper is concerned with the development of a general methodology for identifying mechanical sources from prior local information on both their nature and location over the studied structure. For this purpose, the formulation of the identification problem is derived from the Bayesian statistics, that provides a flexible way to account for local a priori on the distribution of sources. Practically, the resulting optimization problem can be seen as a group generalized Tikhonov regularization, that is solved in an iterative manner. The main features of the proposed identification method are illustrated with both numerical and experimental examples. In particular, it is shown that properly exploiting the local spatial information drastically improves the quality of the source identification.

Keywords: Inverse problem, Force identification, Group generalized Tikhonov regularization, Local priors.
\end{abstract}

\section{Introduction}

In an industrial context, the identification of sources exciting a structure remains an important topic. It can serve for instance to control vibration at

\footnotetext{
${ }^{*}$ Corresponding author. E-mail address : mathieu.aucejo@cnam.fr 
sources, identify excitation sources in unmeasured areas, estimate reaction forces at boundaries or establish excitation models to numerically predict the response of structures. However, the identification of mechanical sources from vibration measurements is known to be an ill-posed inverse problem, meaning that the existence of a unique stable solution is not guaranteed. A classical approach to bypass this difficulty consists in including in the formulation of the inverse problem some prior information on the noise and the spatial distribution of sources to identify to constrain the space of solutions. For this purpose, the Bayesian formalism is generally adopted, since it offers a rigorous mathematical framework that allows combining both probabilistic and mechanical data. Among all the methods used for mechanical source identification and deriving from the Bayesian statistics, the Tikhonov regularization is certainly the most popular $[1,2,3,4]$. Unfortunately, this regularization leads to a systematic smoothing of regularized solutions [5], which is not a desirable effect when a localized source has to be identified. To remedy this problem, several approaches also based on the Bayesian inference have been proposed. In particular, it is useful to cite the work of Renzi et al. [5, 6] in which they apply a Richardson-Lucy deconvolution. From the Bayesian standpoint, this approach consists in assuming a Poisson noise model and a uniform distribution for the source vector to identify [7]. Practically, this leads to impose a positivity constraint on the excitation field to identify, which implies the loss of the phase relationships between the identified sources. Another technique, based on the use of generalized Gaussian laws to reflect prior information on the noise and the sources to identify, has been proposed in [8]. This approach has the advantage to alleviate the 
positivity constraint and to be flexible enough to identify sources of various types. However, in the procedures described above, the a priori on the spatial distribution of the sources remains global. Incidentally, poor identification can be obtained, if the actual excitation field combines both localized and distributed sources, since the a priori has to reflect a compromise between two contradictory distributions. Finally, it is worth citing the work of Zhang et al. [9] in which they propose to solve the Bayesian force reconstruction problem with an uncertain model from Monte Carlo Markov Chain (MCMC) methods. Such an approach has the merit of providing a credible interval on the reconstructed force, which allows assessing the robustness of the identified solution.

The present paper aims at showing that one's prior knowledge of the system can be incorporated to aid the identification process. More precisely, the main objective is to provide a flexible identification methodology, based on Bayesian statistics, able to take advantage of prior local information available on both the nature and the location of excitation sources. To this end, the structure is divided into several identification regions, in which it is assumed that local priors on the nature of the sources follow generalized Gaussian distributions. In doing so, prior information can be accurately adapted to a prescribed zone of the studied structure and, therefore, one can fully exploit the spatial information that is generally available in practical situations. From a mathematical point of view, the solution of the problem is defined as the maximum a posteriori estimate of the posterior distribution. Practically, one seeks the solution of the dual minimization problem, which is solved in an 
iterative manner using a Generalized Iteratively Reweighted Least-Squares (GIRLS) algorithm [8, 10].

To highlight the main features of the proposed methodology, the present article is divided into four parts. In section 2, the formulation of the proposed source identification problem is introduced. In section 3, the resolution of the resulting source identification problem is presented. As mentioned previously, the resolution is performed using the GIRLS algorithm described in [8]. In order to make this paper clearer and more self-contained, the GIRLS algorithm is detailed by introducing further insights regarding the choice of the tuning parameters involved in its definition. Finally, the validity of the proposed methodology is illustrated both numerically and experimentally in sections 4 and 5. In particular, the proposed validations reveal that using local instead of global priors leads to more accurate identifications when a structure is excited by sources having different spatial distributions.

\section{Formulation of the Bayesian source identification problem}

This section aims at presenting the derivation of the formulation of the identification problem and introducing the main tuning parameters involved in the proposed formulation.

\subsection{Problem description}

Let us consider the situation where a vibration field $\mathbf{X}$ is caused by an unknown excitation field $\mathbf{F}$. If the structure is linear, its dynamic behavior is completely defined by the transfer functions matrix $\mathbf{H}$, relating the vibration

field $\mathbf{X}$ to the excitation field $\mathbf{F}$. Practically, the vibration field $\widetilde{\mathbf{X}}$, measured 
over the surface of a structure, is biased by two independent contributions. The first one is related to measurement errors, since the transducers used to perform measurements are imperfect. This contribution is generally modeled by an additive noise. The second one is related to modeling errors, which reflect that the model used to determine the transfer functions matrix $\mathbf{H}$ is imperfect. If modeling errors are supposed small enough, it is reasonable to incorporate this bias in the additive noise [9].

As a result, the measured vibration field $\widetilde{\mathbf{X}}$ is obtained from the following direct formulation:

$$
\widetilde{\mathbf{X}}=\mathbf{H F}+\mathbf{N}
$$

where the transfer functions matrix $\mathbf{H}$, the excitation field $\mathbf{F}$ and the global noise $\mathbf{N}$ are fully determined.

Unlike to the direct problem, the structural source identification problem consists in estimating the unknown excitation field $\mathbf{F}$ acting on a structure from the vibration field $\widetilde{\mathbf{X}}$ measured on its surface only. In other words, the noise $\mathbf{N}$ is another unknown of the inverse problem. To deal with such a problem efficiently, the Bayesian paradigm is well adapted and is adopted in this work.

The Bayesian paradigm consists in considering all the parameters of the problem as random variables. Consequently, it is an uncertainty model, in which the uncertainty on each parameter is modeled by a probability distribution, describing the state of knowledge or the a priori on this parameter. From the mathematical standpoint, the formulation of the Bayesian source 
identification problem relies on the Bayes' rule:

$$
p(\mathbf{F} \mid \widetilde{\mathbf{X}}) \propto p(\widetilde{\mathbf{X}} \mid \mathbf{F}) p(\mathbf{F}),
$$

where:

- $p(\mathbf{F} \mid \widetilde{\mathbf{X}})$ is the posterior probability distribution, representing the probability of observing $\mathbf{F}$ given the measured vibration field $\widetilde{\mathbf{X}}$. In other words, it represents what is known on the excitation field after making vibration measurements;

- $p(\widetilde{\mathbf{X}} \mid \mathbf{F})$ is the likelihood function, representing the probability of measuring $\widetilde{\mathbf{X}}$ given an excitation field $\mathbf{F}$. It reflects the uncertainty related to the measurement of the vibration field, which is itself related to the noise;

- $p(\mathbf{F})$ is the prior probability distribution, representing the prior knowledge of the excitation field before measuring the vibration field.

From the Bayes' rule, one seeks the most probable excitation field $\widehat{\mathbf{F}}$ given the measured vibration field $\widetilde{\mathbf{X}}$. Such a solution is a point estimate of F corresponding to a mode of the posterior probability distribution. Consequently, the solution of the identification problem is sought as the maximum a posteriori estimate, that is:

$$
\widehat{\mathbf{F}}=\underset{\mathbf{F}}{\operatorname{argmax}} p(\mathbf{F} \mid \widetilde{\mathbf{X}})=\underset{\mathbf{F}}{\operatorname{argmax}} p(\widetilde{\mathbf{X}} \mid \mathbf{F}) p(\mathbf{F}) .
$$

Practically, it is generally easier to find the solution of the following dual minimization problem:

$$
\widehat{\mathbf{F}}=\underset{\mathbf{F}}{\operatorname{argmin}}-\ln p(\mathbf{F} \mid \widetilde{\mathbf{X}})=\underset{\mathbf{F}}{\operatorname{argmin}}-\ln p(\widetilde{\mathbf{X}} \mid \mathbf{F})-\ln p(\mathbf{F}) .
$$


From Eqs. (2)-(4), it is clear that the more prior information reflects the nature of the noise and the sources to identify, the more the posterior probability is high. From this observation, it is expected that proper results can be obtained if the likelihood function and the prior probability distribution are properly chosen, since the identification process gives the most probable solution given prior information on the noise and the nature of the sources to identify.

\subsection{Choice of the likelihood function and prior probability distribution}

As suggested in the previous section, the quality of the source identification is closely related to the choice of the likelihood function and prior probability distribution. That is why, this section aims at explaining the choices made in this paper to have a closed-form expression of the identification problem, that allows properly reflecting prior information on the noise and the sources to identify.

\subsubsection{Choice of the likelihood function}

As mentioned in section 2.1, the likelihood function reflects the uncertainty related to the measurement of the vibration field $\widetilde{\mathbf{X}}$. By definition [see Eq. (1)], this uncertainty is related to the noise $\mathbf{N}$. Consequently, the likelihood function can be rewritten under the following form:

$$
p(\widetilde{\mathbf{X}} \mid \mathbf{F})=p(\widetilde{\mathbf{X}}-\mathbf{H F} \mid \mathbf{N}),
$$

meaning that the likelihood function can be seen as the probability of obtain-

ing $\widetilde{\mathbf{X}}-\mathbf{H F}=\mathbf{0}$ given the noise $\mathbf{N}$. In other words, the likelihood function measures the fidelity of the vibration model, corresponding to the noiseless 
vibration field $\mathbf{X}=\mathbf{H F}$, to the measured data. As a result, the likelihood function is also written:

$$
p(\widetilde{\mathbf{X}} \mid \mathbf{F})=p(\mathbf{N}),
$$

where $p(\mathbf{N})$ is the prior probability distribution of the noise, representing the a priori of the experimenter on the nature of the noise inducing a discrepancy between the measured vibration field and its noiseless estimate before any measurement of the vibration field.

If the noise is supposed spatially white (meaning that the components of $\mathbf{N}$ are independent and identically distributed random variables) and due to multiple independent causes, then the prior probability distribution of the noise can be represented by a multivariate normal distribution with zero mean and variance $\alpha^{2}$ :

$$
p(\mathbf{N}) \propto \exp \left[-\frac{1}{2} \frac{\|\mathbf{N}\|_{2}^{2}}{\alpha^{2}}\right],
$$

where $\|\bullet\|_{2}$ is the $\mathrm{L}_{2}$-norm, also known as the Euclidean norm.

It can be inferred from Eqs. (1), (6) and (7) that the likelihood function is written:

$$
p(\widetilde{\mathbf{X}} \mid \mathbf{F}) \propto \exp \left[-\frac{1}{2} \frac{\|\widetilde{\mathbf{X}}-\mathbf{H F}\|_{2}^{2}}{\alpha^{2}}\right] .
$$

Finally, it is worth mentioning that in practical situations the noise may not be strictly white or even Gaussian. However, assuming a spatially Gaussian white noise allows limiting the number of hyperparameters of the Bayesian model. Consequently, this hypothesis can seem coarse or indecorous, but it is actually relevant as highlighted by the experimental results 
presented in the next of the paper.

\subsubsection{Choice of the prior probability distribution}

The prior probability distribution reflects the uncertainty related to the unknown excitation field $\mathbf{F}$. Consequently, it can be seen as the a priori of the experimenter on the sources to identify. To take advantage of local information available a priori on the nature and the spatial distribution of sources, it is supposed that the structure is excited in $N$ different regions by uncorrelated excitations of various types (localized or distributed). This allows considering the local excitation fields $\mathbf{F}_{\mathbf{i}}$ as independent random vectors and thus writing the prior probability distribution as the product of local prior probability distributions $p\left(\mathbf{F}_{\mathbf{i}}\right)$, that is:

$$
p(\mathbf{F})=\prod_{i=1}^{N} p\left(\mathbf{F}_{\mathbf{i}}\right),
$$

where $p\left(\mathbf{F}_{\mathbf{i}}\right)$ reflects the prior knowledge of the nature of the sources in the zone i.

Furthermore, it is assumed that the components of each local excitation vector $\mathbf{F}_{\mathbf{i}}$ are independent and identically distributed random variables (i.e. uncorrelated but having the same probability distribution) following a generalized Gaussian distribution with zero mean. As a consequence, each local excitation field follows a multivariate generalized Gaussian distribution with zero mean. From the mathematical standpoint, the local prior probability distributions are thus written:

$$
p\left(\mathbf{F}_{\mathbf{i}}\right) \propto \exp \left[-\frac{1}{q_{i}} \frac{\left\|\mathbf{L}_{\mathbf{i}} \mathbf{F}_{\mathbf{i}}\right\|_{q_{i}}^{q_{i}}}{\beta_{i}^{q_{i}}}\right],
$$


where:

- $q_{i}$ is the shape parameter of the distribution in the zone i. Its value is defined in the interval ]0, $+\infty[$;

- $\|\bullet\|_{q_{i}}$ is the $\mathrm{L}_{\mathrm{q}_{\mathrm{i}}}$-norm or quasi-norm, if $q_{i} \geq 1$ and $q_{i}<1$ respectively;

- $\beta_{i}$ is the scale parameter of the distribution, which defines the dispersion of the distribution around the mean. It is therefore a generalized measure of the variance of the distribution;

- $\mathbf{L}_{\mathbf{i}}$ is a differentiation matrix controlling the regularity of the solution in the zone $\mathrm{i}$.

It should be noted that the choice of a multivariate generalized Gaussian distribution allows a great flexibility for describing prior knowledge of the sources to identify as it will be made clearer in section 3.2.1. As for the assumption of a Gaussian white noise, the hypothesis of independence used to derive the prior probability distribution can be discussed. Actually, this hypothesis has been introduced here because the correlation between each component of the force vector is difficult to assess a priori. However, this assumption is typically violated when one tries to identify excitation forces and forces at boundaries. In this particular situation, forces at boundaries are functionally dependent on the excitation forces. From a theoretical standpoint, Tikhonov-like approaches are known in Machine Learning and Data Mining as naive Bayes classifiers. In these fields, it has been shown that naive Bayes classifiers works well not only when the parameters involved in the formulation are completely independent, but also when the parame- 
ters are functionally dependent [11]. For all the aforementioned reasons, the hypothesis of independence is relevant.

\subsection{Practical form of the identification problem}

In order to derive the practical form of the identification problem, Eqs. (8) and (10) are introduced into Eq. (4). In doing so, one obtains the general formulation of the identification problem given below:

$$
\widehat{\mathbf{F}}=\underset{\mathbf{F}}{\operatorname{argmin}} \frac{1}{2} \frac{\|\widetilde{\mathbf{X}}-\mathbf{H F}\|_{2}^{2}}{\alpha^{2}}+\sum_{i=1}^{N} \frac{1}{q_{i}} \frac{\left\|\mathbf{L}_{\mathbf{i}} \mathbf{F}_{\mathbf{i}}\right\|_{q_{i}}^{q_{i}}}{\beta_{i}^{q_{i}}} .
$$

At this stage, $2 N+1$ parameters, namely $\left(q_{i}, \beta_{i}, \alpha\right)$, are necessary to mathematically describe prior information on the noise and the sources to identify. However, it can be emphasized that the parameters $q_{i}$ and $\beta_{i}$ play a similar role in this formulation. Therefore, only one set of parameters, $q_{i}$ or $\beta_{i}$, can be used. In the present paper, it has been chosen to work with the shape parameter $q_{i}$, while setting the values of the parameters $\beta_{i}^{q_{i}}$ to a unique constant value $\underline{\beta}$. Taking this observation into account, one finally gets the final form of the identification problem, which is a group generalized Tikhonov regularization :

$$
\widehat{\mathbf{F}}=\underset{\mathbf{F}}{\operatorname{argmin}} \frac{1}{2}\|\widetilde{\mathbf{X}}-\mathbf{H F}\|_{2}^{2}+\lambda \sum_{i=1}^{N} \frac{1}{q_{i}}\left\|\mathbf{L}_{\mathbf{i}} \mathbf{F}_{\mathbf{i}}\right\|_{q_{i}}^{q_{i}},
$$

where $\lambda=\alpha^{2} / \underline{\beta}$ is the so-called regularization parameter, that controls the trade-off between prior information on the noise and prior information on the solution.

Before going any further, it could be interesting to discuss the frequency limitations of the proposed identification procedure. Actually, for a fully 
determined problem, the limitations are not related to the formulation of the inverse problem itself, but to the measurement or the calculation of the transfer functions matrix $\mathbf{H}$ and the measurement of the vibration field $\widetilde{\mathbf{X}}$. Regarding the calculation of $\mathbf{H}$, the frequency limitation is related to the model of the structure. If the model is purely analytic, then the limit is related to the assumptions made to establish the mechanical model. If the model is obtained by FEM, then the limit is fixed by the assumptions made to derive the mechanical model and the mesh used to discretize the structural domain (generally built using 6 nodes per wavelength). Regarding the measurement of the vibration field, the frequency limitation is mainly related to the bandwidths and the resolution of the transducers.

It can also be noticed that the proposed formulation includes the standard Tikhonov regularization, since the latter is recovered if $q_{i}=2$ for all $\mathrm{i} \in[1, N]$ in Eq. (12). Furthermore, it is emphasized that the selected zones can cover only a part of the whole structure, implying that no a priori on the spatial distribution of sources is assumed in non-selected zones. In these particular regions, the identification is therefore only based on the fitting to the experimental data, i.e. on prior information on the noise. Finally, it is worth noting that the robustness of the proposed method against modeling and measurement uncertainties can not be assessed, since the proposed formulation provides a point estimate of the force vector to identify. Nevertheless, the proposed methodology allows obtaining consistent identifications as it will be shown in sections 4 and 5 . 


\section{Resolution of the source identification problem}

Although the proposed formulation is highly flexible to describe prior knowledge of the sources to identify, its solution has generally no closed-form expression. That is why, the identification problem is solved using an iterative algorithm, which is a generalized version of the Iteratively Reweighted LeastSquares algorithm [8, 10, 12]. The proposed algorithm has the advantage to be easy to implement and general enough to cope with a wide range of configurations depending on the values of $q_{i}$. In the next of the paper, it is referred to as Generalized Iteratively Reweighted Least-Squares (GIRLS) algorithm.

\subsection{General principle}

The core idea of the GIRLS algorithm consists in replacing the direct resolution of the minimization problem [see Eq. (12)] by an equivalent iterative process having an explicit solution at each iteration. For this purpose, one has to notice that the $\mathrm{L}_{\mathrm{q}}$-norm to the power of $q$ can be rewritten as a squared weighted $\mathrm{L}_{2}$-norm as it is shown in Eq. (13):

$$
\forall x_{n}, \forall q, \frac{1}{q}\|\mathbf{x}\|_{q}^{q}=\frac{1}{q} \sum_{n}\left|x_{n}\right|^{q}=\frac{1}{2} \sum_{n} w\left(x_{n}\right)\left|x_{n}\right|^{2}=\frac{1}{2}\left\|\mathbf{W}^{1 / 2} \mathbf{x}\right\|_{2}^{2},
$$

where $w\left(x_{n}\right)=\frac{2}{q}\left|x_{n}\right|^{q-2}$ is the weighting coefficient and $\mathbf{W}=\operatorname{diag}\left[w\left(x_{1}\right), \ldots, w\left(x_{n}\right)\right]$ is the corresponding definite positive weighting matrix.

Since the weighting matrix depends on $\mathbf{x}$, the equality given in Eq. (13) cannot be verified directly, which justifies the use of an iterative algorithm to obtain the solution of the identification problem. As part of an iterative 
process, the aim is thus to find the solution $\mathbf{x}^{(k+1)}$ at iteration $\mathrm{k}+1$ from the solution $\mathbf{x}^{(k)}$ at iteration $\mathrm{k}$ by setting $w\left(x_{n}\right)=w\left(x_{n}^{(k)}\right)$, so as to recover the identity given by Eq. (13) when the iterative process has converged. When applying this idea to the proposed formulation, it can be inferred that the excitation field $\widehat{\mathbf{F}}^{(k+1)}$ at iteration $\mathrm{k}+1$ is solution of the following minimization problem:

$$
\widehat{\mathbf{F}}^{(k+1)}=\underset{\mathbf{F}}{\operatorname{argmin}} \frac{1}{2}\|\widetilde{\mathbf{X}}-\mathbf{H F}\|_{2}^{2}+\frac{\lambda^{(k+1)}}{2} \sum_{i=1}^{N}\left\|\mathbf{W}_{\mathbf{i}}^{(k)^{1 / 2}} \mathbf{L}_{\mathbf{i}} \mathbf{F}_{\mathbf{i}}\right\|_{2}^{2},
$$

where $\lambda^{(k+1)}$ is the regularization parameter at iteration $\mathrm{k}+1$.

For numerical purposes, the definition of the weighting matrix $\mathbf{W}_{\mathbf{i}}^{(k)}$ is slightly modified compared to that given in Eq. (13), since it is defined such that:

$$
\mathbf{W}_{\mathbf{i}}^{(k)}=\operatorname{diag}\left[\frac{2}{q_{i}} T_{\epsilon}\left(\mathbf{L}_{\mathbf{i}} \mathbf{F}_{\mathbf{i}}^{(k)}\right)\right]
$$

with:

$$
T_{\epsilon}\left(x_{j}^{(k)}\right)= \begin{cases}\left|x_{j}^{(k)}\right|^{q_{i}-2} & \text { if }\left|x_{j}^{(k)}\right|>\epsilon_{i}^{(k)} \\ \epsilon_{i}^{(k)^{q_{i}-2}} & \text { if }\left|x_{j}^{(k)}\right| \leq \epsilon_{i}^{(k)}\end{cases}
$$

where $x_{j}^{(k)}$ is the $\mathrm{j}$-th component of the vector $\mathbf{x}^{(k)}$ and $\epsilon_{i}^{(k)}$ is a small real positive number acting as a damping parameter. It allows avoiding infinite weights when $\left|x_{j}^{(k)}\right| \rightarrow 0$ and $q_{i}<2$.

For implementation purposes, it should be emphasized that Eq. (14) can be more concisely expressed by introducing two matrices, $\overline{\mathbf{W}}^{(k)}$ and $\overline{\mathbf{L}}$, defined 
as follows:

$$
\overline{\mathbf{W}}^{(k)}=\left(\begin{array}{rrr}
\mathbf{W}_{\mathbf{1}}^{(k)} & & \mathbf{0} \\
& \ddots & \\
\mathbf{0} & & \mathbf{W}_{\mathbf{N}}^{(k)}
\end{array}\right)
$$

and

$$
\overline{\mathbf{L}}=\left(\begin{array}{ccc}
\mathbf{L}_{1} & & \mathbf{0} \\
& \ddots & \\
\mathbf{0} & & \mathbf{L}_{\mathbf{N}}
\end{array}\right),
$$

if the selected zones entirely cover the studied structure. In all other cases,

$$
\overline{\mathbf{L}}=\left(\begin{array}{cccc}
\mathbf{L}_{1} & & \mathbf{0} & \mathbf{0} \\
& \ddots & & \vdots \\
0 & & \mathbf{L}_{\mathbf{N}} & \mathbf{0}
\end{array}\right) .
$$

Introducing $\overline{\mathbf{W}}^{(k)}$ and $\overline{\mathbf{L}}$ into Eq.(14), it readily comes that:

$$
\widehat{\mathbf{F}}^{(k+1)}=\underset{\mathbf{F}}{\operatorname{argmin}} \frac{1}{2}\|\widetilde{\mathbf{X}}-\mathbf{H F}\|_{2}^{2}+\frac{\lambda^{(k+1)}}{2}\left\|\overline{\mathbf{W}}^{(k)^{1 / 2}} \overline{\mathbf{L}} \mathbf{F}\right\|_{2}^{2} .
$$

\subsection{Choice of the tuning parameters}

According to Eqs. (14), (15) and (16), $2 N+1$ tuning parameters are involved in the resolution of the identification problem, namely the shape parameters of the distribution $q_{i}$, the regularization parameter $\lambda^{(k+1)}$ and the damping parameters $\epsilon_{i}^{(k)}$. In the following subsections, the selection of each parameter is detailed.

\subsubsection{Choice of the shape parameters}

To properly choose the value of the shape parameter in each zone, one has to keep in mind that the shape of the distribution or equivalently the shape 
of the penalty function, i.e. $\|\mathbf{x}\|_{q_{i}}^{q_{i}}$, has a major influence on the solution of the identification problem [see Fig. 1]. Indeed, as shown in Fig. 1b, it is clear that the smaller $q_{i}$ is, the larger is the weight on small values of $\mathbf{x}$. On the contrary, for large values of $\mathbf{x}$, the smaller $q_{i}$ is, the smaller is the weight on these values. This difference in the weighting behavior for small and large values of $\mathbf{x}$ can be directly observed in the solutions obtained, since for $q_{i} \leq 1$ the solution will tend to have only a few non-zero values, while for $q_{i}=2$ the solution will tend to have only a few very small values.

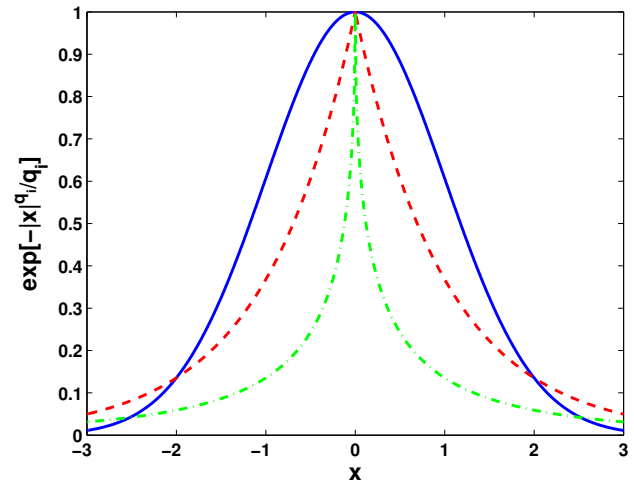

(a)

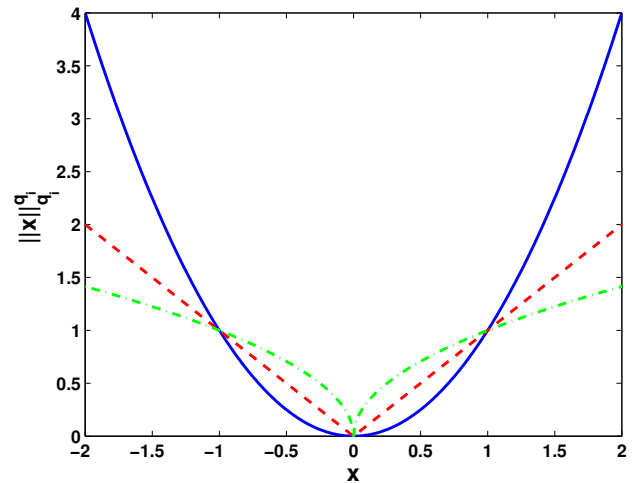

(b)

Figure 1: Selection of the shape parameter $q_{i}$ - (a) Probability distribution $\exp \left[-\frac{\|\mathbf{x}\|_{q_{i}}^{q_{i}}}{q_{i} \beta_{i}^{q_{i}}}\right]$ $\left(\beta_{i}^{q_{i}}=1\right)$ and (b) Corresponding penalty function $\|\mathbf{x}\|_{q_{i}}^{q_{i}}$ for $(-) q_{i}=2,(--) q_{i}=1$ and $(-\cdot-) q_{i}=0.5$

From this observation, it can be inferred that $q_{i}$ can be set to 2 if the solution sought is supposed to be distributed [13], while $q_{i}$ can be chosen equal to or less than 1 if the solution is supposed to be localized [14, 15]. This rule of thumb essentially holds if the differentiation matrices $\mathbf{L}_{\mathbf{i}}$ correspond to the identity matrix. Indeed, if ones tries to identify a piecewise 
continuous excitation field in a zone i, one has to promote a solution for which the first derivative contains only a few non-zero values. In this situation, the differentiation matrix differs from the identity matrix. As an example, for a one dimensional structure, it is then recommended to choose $q_{i} \leq 1$ and use a differentiation matrix of the form:

$$
\mathbf{L}_{\mathbf{i}}=\left(\begin{array}{cccccc}
-1 & 1 & & & & \\
& -1 & 1 & & & \\
& & & \ddots & & \\
& & & & -1 & 1
\end{array}\right)
$$

\subsubsection{Choice of the regularization parameter}

The value of the regularization parameter can be chosen automatically from various selection methods, such as the Morozov's discrepancy principle [16], the Generalized Cross Validation [17], the Bayesian estimator [18] or the L-curve principle [19]. In the present paper, the regularization parameter $\lambda^{(k)}$ is obtained using the L-curve principle at each iteration.

For the sake of completeness, it can be reminded that the L-curve principle consists in plotting, in a log-log scale, the $\mathrm{L}_{2}$-norm of the regularization term (i.e. $\left\|\overline{\mathbf{W}}^{(k)^{1 / 2}} \overline{\mathbf{L}} \mathbf{F}\right\|_{2}$ ) versus the $\mathrm{L}_{2}$-norm of the data fidelity term (i.e. $\|\widetilde{\mathbf{X}}-\mathbf{H F}\|_{2}$ ) for various values of the regularization parameter. Generally, the resulting curve exhibits an L-shape. The optimal value of the regularization parameter is the value for which the curvature of the L-curve is maximal, i.e. corresponding to the corner of the L-curve. To illustrate the selection of the regularization parameter from the L-curve principle, an example extracted from the experimental validation presented in section 5 is proposed in Fig. 2. 


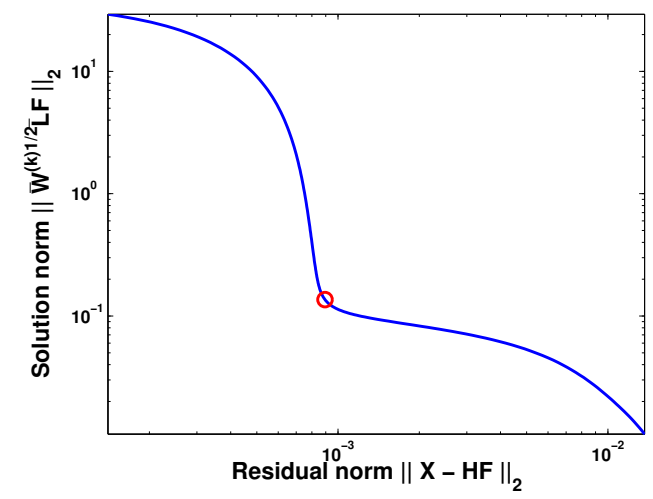

(a)

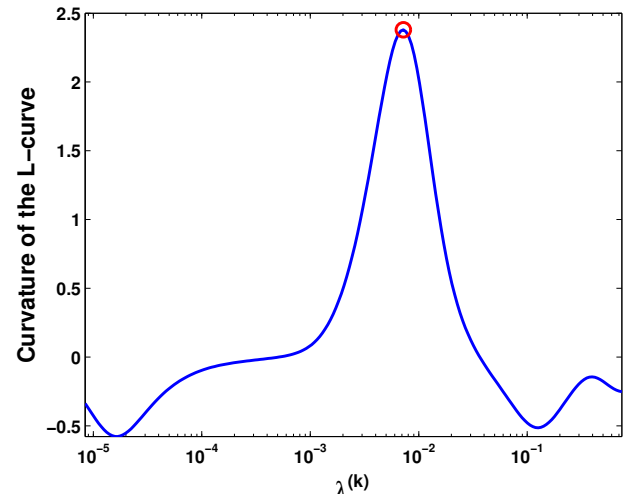

(b)

Figure 2: Illustration of the L-curve principle - (a) Plot of the L-curve and (b) Curvature of the L-curve versus regularization parameter $\lambda^{(k)}-(\circ)$ Location of the optimal regularization parameter

However, the computation of the regularization parameter at each iteration is intensive. Accordingly, it is interesting to determine whether a unique value can be used throughout the iterative process. As pointed out in [8], a series of numerical experiments has shown that using $\lambda^{(k)}=\lambda^{(0)}$ allows obtaining similar results to those obtained from updated $\lambda^{(k)}$ within a comparable number of iterations. The logical outcome of this numerical study is that the choice of the regularization parameter can be performed once for all during the initialization step of the iterative process.

\subsubsection{Choice of the damping parameters}

The damping parameter $\epsilon_{i}^{(k)}$ can be chosen automatically for each zone from the cumulative histogram of $\left|\mathbf{L}_{\mathbf{i}} \widehat{\mathbf{F}}_{\mathbf{i}}^{(k)}\right|[20]$, meaning that the value of the damping parameter is chosen so that a fixed percentage $p$ of the values of 
$\left|\mathbf{L}_{\mathbf{i}} \widehat{\mathbf{F}}_{\mathbf{i}}^{(k)}\right|$ are less than or equal to $\epsilon_{i}^{(k)}$ [see Fig. 3]. From our own numerical tests, it has been found that using $p=5 \%$ leads to satisfying results.

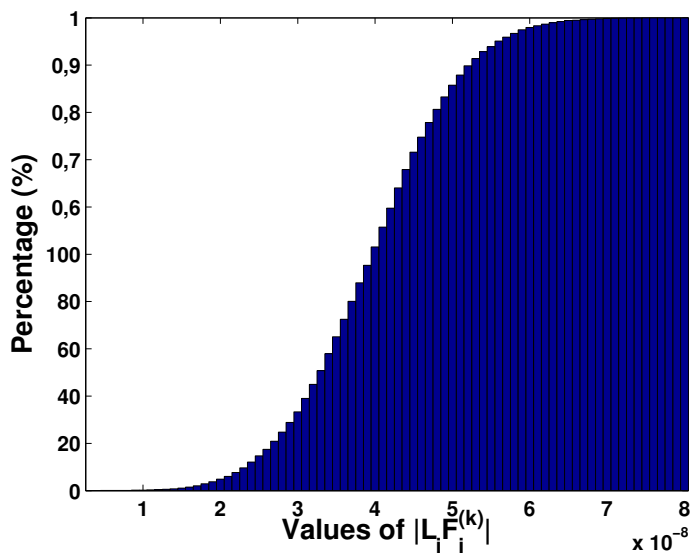

Figure 3: Illustration of the choice of the damping parameter - Cumulative histogram of $\left|\mathbf{L}_{\mathbf{i}} \widehat{\mathbf{F}}_{\mathbf{i}}^{(k)}\right|$

As for the regularization parameter, accurate solutions can be obtained using a fixed value of the damping parameter. Indeed, from a set of numerical experiments, it has been noticed that using the value computed during the initialization step throughout the iterative process (i.e. $\epsilon_{i}^{(k)}=\epsilon^{(0)}$ ) has no significant influence on both the quality and the time-performance of the algorithm.

\subsection{Choice of the initial guess and stopping criterion}

Since the proposed resolution algorithm is iterative, another crucial issue is the choice of the initial guess as well as that of the stopping criterion. 


\subsubsection{Choice of the initial guess}

Choosing a good initial guess is one of the keys for a successful identification, since the proposed formulation is non-convex when $q_{i}<1$. In such a situation, the existence of a unique minimizer is not guaranteed. Conse-

quently, the identified solution $\widehat{\mathbf{F}}$ strongly depends on the initial guess $\widehat{\mathbf{F}}^{(0)}$. A good initial guess can be defined as a coarse solution of the problem, easy to compute, but sufficiently close to the solution sought to ensure the convergence of the iterative process. A possible choice that fulfills this requirement is the solution of the standard Tikhonov regularization, that is:

$$
\widehat{\mathbf{F}}^{(0)}=\underset{\mathbf{F}}{\operatorname{argmin}} \frac{1}{2}\|\widetilde{\mathbf{X}}-\mathbf{H F}\|_{2}^{2}+\frac{\lambda^{(0)}}{2}\|\mathbf{F}\|_{2}^{2}
$$

\subsubsection{Choice of the stopping criterion}

As an iterative process, the GIRLS algorithm requires the definition of a practical test that allows determining when to stop the algorithm. In the present paper, the stopping criterion is related to the relative variation of the functional $J\left(\widehat{\mathbf{F}}^{(k)}\right)=\frac{1}{2}\left\|\widetilde{\mathbf{X}}-\mathbf{H} \widehat{\mathbf{F}}^{(k)}\right\|_{2}^{2}+\frac{\lambda^{(k)}}{2}\left\|\overline{\mathbf{W}}^{(k-1)^{1 / 2}} \overline{\mathbf{L}}^{(k)}\right\|_{2}^{2}$ between two successive iterations. As classically done in the literature, the relative variation $\delta$ of the functional $J$ is defined such that:

$$
\delta=\frac{\left|J\left(\widehat{\mathbf{F}}^{(k+1)}\right)-J\left(\widehat{\mathbf{F}}^{(k)}\right)\right|}{J\left(\widehat{\mathbf{F}}^{(k)}\right)} .
$$

From there, the iterative process is stopped when the relative variation $\delta$ is less than or equal to some tolerance. Experimentally, it has been found that setting the tolerance to $10^{-8}$ allows obtaining consistent identified solutions, while preserving the time-performance of the GIRLS algorithm. 


\subsection{Summary}

To clearly highlight each step of the resulting iterative process, a comprehensive overview of the proposed version of the GIRLS algorithm is given in table 1.

Table 1: Generic GIRLS algorithm

\section{Proposed GIRLS Algorithm}

Inputs: Transfer functions matrix: $\mathbf{H}$, Measured vibration field $\widetilde{\mathbf{X}}$,

Selected zones $\{i\}$, Differentiation matrix for each selected zones: $\mathbf{L}_{\mathbf{i}}$,

Tolerance: tol

Output: Estimated force vector: $\widehat{\mathbf{F}}$

Initialization: Compute $\lambda^{(0)}$ from the L-curve principle

Compute $\widehat{\mathbf{F}}^{(0)}$ from Eq. $(22)$

Compute $\epsilon^{(0)}$ from the cumulative histogram of $\left|\widehat{\mathbf{F}}^{(0)}\right|$

Initialize $\delta$ to 1

\section{Iteration:}

while $\delta>$ tol

Compute $\mathbf{W}_{\mathbf{i}}^{(k)}$ from Eq. (15) using $\epsilon_{i}^{(k)}=\epsilon^{(0)}$

Construct $\overline{\mathbf{W}}^{(k)}$ from Eq. (17)

Construct $\overline{\mathbf{L}}$ from Eq. (18) or Eq.(19)

Compute $\widehat{\mathbf{F}}^{(k+1)}$ from Eq. (14) using $\lambda^{(k+1)}=\lambda^{(0)}$

Update $\delta$ using Eq. (23)

$k \leftarrow k+1$

end

return $\widehat{\mathbf{F}} \leftarrow \widehat{\mathbf{F}}^{(k)}$ 


\section{Numerical validation}

In general, forces and moments can be applied on a structure or exists at boundaries. However, the identification of moments requires a careful attention [4], since rotations are difficult to measure directly. For this reason, the proposed numerical validation is restricted to the identification of forces only, in order to focus on the particular interest of including local prior information in the identification process, which is the originality of the paper.

\subsection{Description of the test case}

In the present numerical validation, one seeks to identify a mechanical point force acting on a thin simply supported steel plate. In such a configuration, the excitation field exhibits two types of spatial distribution over the structure, namely a smooth distribution of the reaction forces at boundaries and a singular distribution around the location of the point force. The main simulation parameters of the proposed test case are listed in table 2 .

The exact vibration displacement field $\mathbf{X}^{\text {exact }}$ is computed from a FE mesh of the plate made up of 567 linear quadrilateral shell elements, assuming that only bending motions are measurable. In practical situations, the exact vibration field is corrupted by noise. This phenomenon is modeled by adding to the exact displacement field an additive Gaussian white noise as suggested by Eq. (1), that is:

$$
\widetilde{\mathbf{X}}=\mathbf{X}^{\text {exact }}+\mathbf{N}
$$

where the noise vector $\mathbf{N}$ is such that [21]:

$$
\mathbf{N}=\boldsymbol{\beta}_{\mathbf{a}} e^{j \boldsymbol{\phi}_{\mathbf{a}}},
$$


Table 2: Simulation parameters

\begin{tabular}{ll}
\hline Parameters & Values \\
\hline Plate length & $L_{x}=2 \mathrm{~m}$ \\
Plate width & $L_{y}=1.5 \mathrm{~m}$ \\
Plate thickness & $h=0.017 \mathrm{~m}$ \\
Young's modulus & $E=2.1 \times 10^{11} \mathrm{~Pa}$ \\
Density & $\rho=7800 \mathrm{~kg} \cdot \mathrm{m}^{-3}$ \\
Structural damping & $\eta=0.01$ \\
Amplitude of the force & $\mathrm{F}_{0}=1 \mathrm{~N}$ \\
Location of the force & $\left(x_{0}, y_{0}\right)=(1.04 \mathrm{~m}, 0.93 \mathrm{~m})$ \\
Studied frequency & $f_{0}=450 \mathrm{~Hz}$ \\
\hline
\end{tabular}

where $\boldsymbol{\beta}_{\mathbf{a}}=p_{n} M^{-1 / 2}\left\|\mathbf{X}^{\text {exact }}\right\|_{2} \mathbf{Z}$ and $\boldsymbol{\phi}_{\mathbf{a}}=\mathbf{U}$, with $p_{n}$ the noise percentage, $M$ the number of measurable dofs, $\mathbf{Z}$ a Gaussian random vector and $\mathbf{U}$ a random vector uniformly distributed over the interval $[0,2 \pi]$. In what follows, the noise percentage $p_{n}$ is set to $2 \%$.

Furthermore, as suggested in [4], a FE model of the plate with free boundary conditions is used to compute the transfer functions matrix $\mathbf{H}$, which is dynamically condensed over the measurable dofs only [8, 22]. The main interest in using free boundary conditions to model dynamic behavior of the plate is to allow the identification of external excitations acting on the structure as well as reaction forces at boundaries. Finally, one can notice that the proposed FE model of the plate is theoretically valid up to $650 \mathrm{~Hz}$, corresponding to the frequency for which the structure is discretized with 6 nodes 
per bending wavelength.

\subsection{Application}

The reference force vector $\mathbf{F}^{\mathrm{ref}}$ is obtained from the transfer functions matrix $\mathbf{H}$ and the exact displacement field $\mathbf{X}^{\text {exact }}$ thanks to the following equation:

$$
\mathbf{F}^{\mathrm{ref}}=\mathbf{H}^{-1} \mathbf{X}^{\text {exact }}
$$

Fig. 4 presents the reference force vector to identify at $450 \mathrm{~Hz}$. As expected, a unit point force appears at $\left(\mathrm{x}_{0}, \mathrm{y}_{0}\right)=(1.04 \mathrm{~m}, 0.93 \mathrm{~m})$ as well as smooth reaction forces at boundaries of the plate.

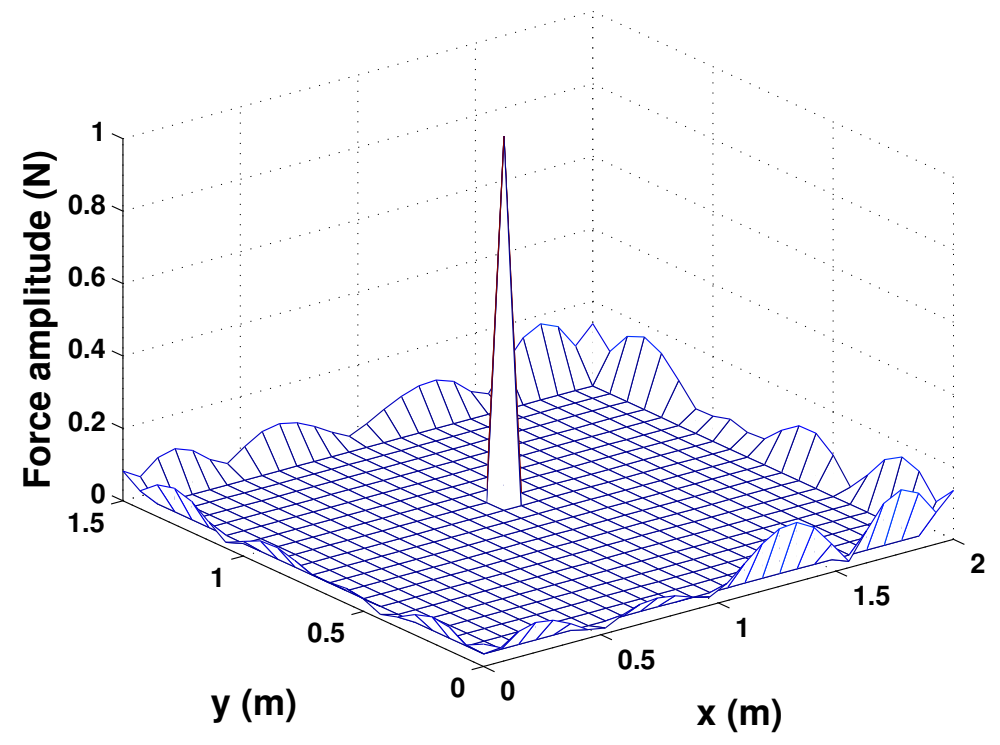

Figure 4: Reference force vector $\mathbf{F}^{\text {ref }}$ at $450 \mathrm{~Hz}$

Replacing naively the exact displacement field by the corrupted one in Eq. (26), one obtains the identified excitation vector $\widehat{\mathbf{F}}$ presented in Fig. 5. 
Obviously, the source identification fails, since the reconstructed excitation field is highly dominated by the noise. This result is easily explained by the ill-conditioning of the transfer function matrix $\mathbf{H}$. In the present case, the condition number of $\mathbf{H}$ is equal to $2 \times 10^{4}$. This indicates the presence of small singular values, which are responsible for the amplification of the terms related to the noise and consequently cause the identification to fail [23].

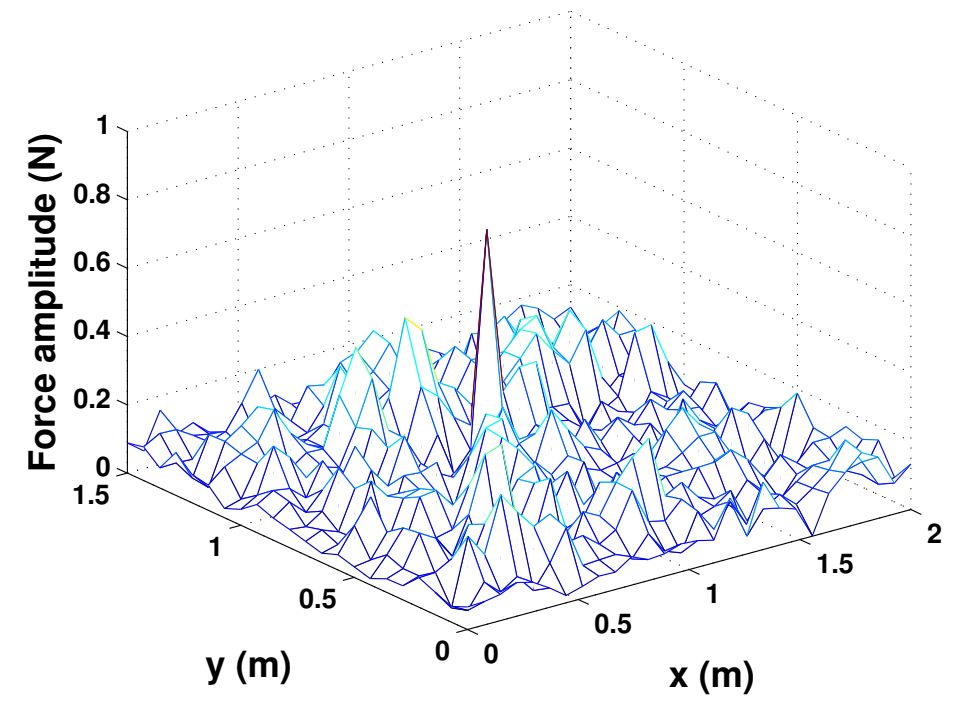

Figure 5: Naive identification of the force vector $\widehat{\mathbf{F}}$ at $450 \mathrm{~Hz}$

As done in Ref. [8], the first idea to stabilize the identification problem consists in using a global a priori on the spatial distribution of sources. In such a configuration, it is clear that a compromise has to be found between the smoothness of the reaction forces at boundaries and the singularity of the point force. Considering explanations given in section 3.2.1, a relevant compromise is obtained after 31 iterations for $q=1.1$ and $\overline{\mathbf{L}}=\mathbf{I}$ as pre- 
sented in Fig. 6. With this value of the shape parameter, the identified force amplitude $\widehat{\mathrm{F}}_{0}$ is equal to $0.72 \mathrm{~N}$ instead of $1 \mathrm{~N}$.

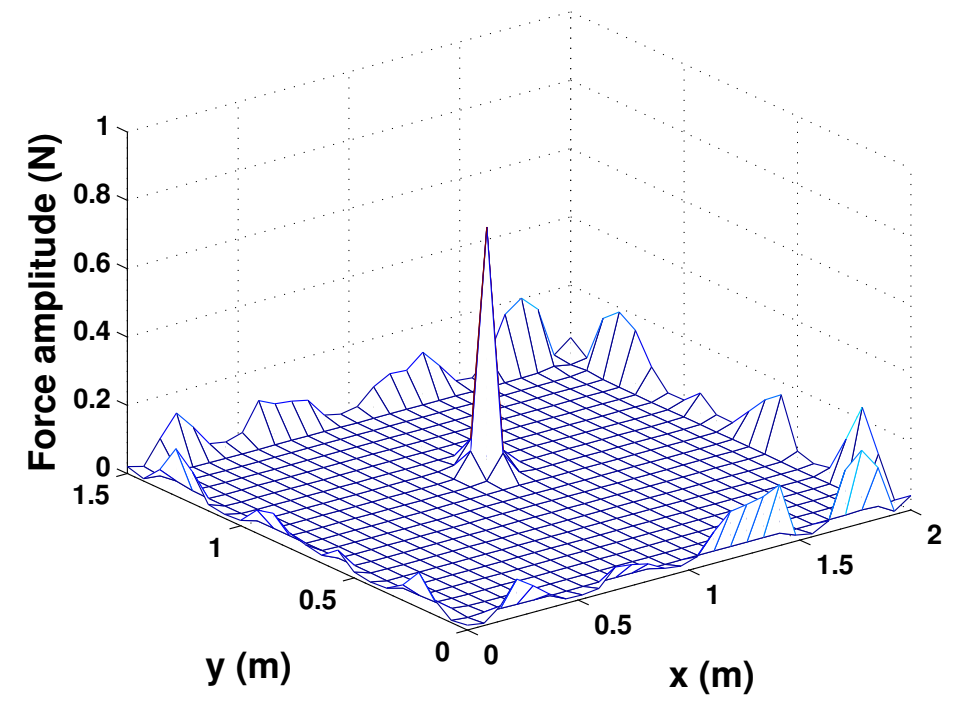

Figure 6: Identification of the force vector $\widehat{\mathbf{F}}$ at $450 \mathrm{~Hz}$ from corrupted data when using a global prior on the spatial distribution of sources $-q=1.1$ and $\overline{\mathbf{L}}=\mathbf{I}$

However, the a priori on the spatial distribution of sources being perfectly adapted neither to localized sources nor to distributed sources, the identified sources do not perfectly match with the actual ones. An even better result can be obtained by using properly our prior knowledge of the location and the nature of the sources acting on the structure. Indeed, as presented in Fig. 4, the force vector to identify is sparse except in the vicinity of the boundaries of the plate. This observation leads to divide the structure into two identification regions: (i) a central region associated to the shape parameter $q_{1}$ and containing the point force only, in which a sparsity-promoting prior is required, and (ii) a region associated to the shape parameter $q_{2}$ and corre- 
sponding to the boundaries of the plate, in which a prior promoting smooth solutions has to be employed [see Fig. 7].

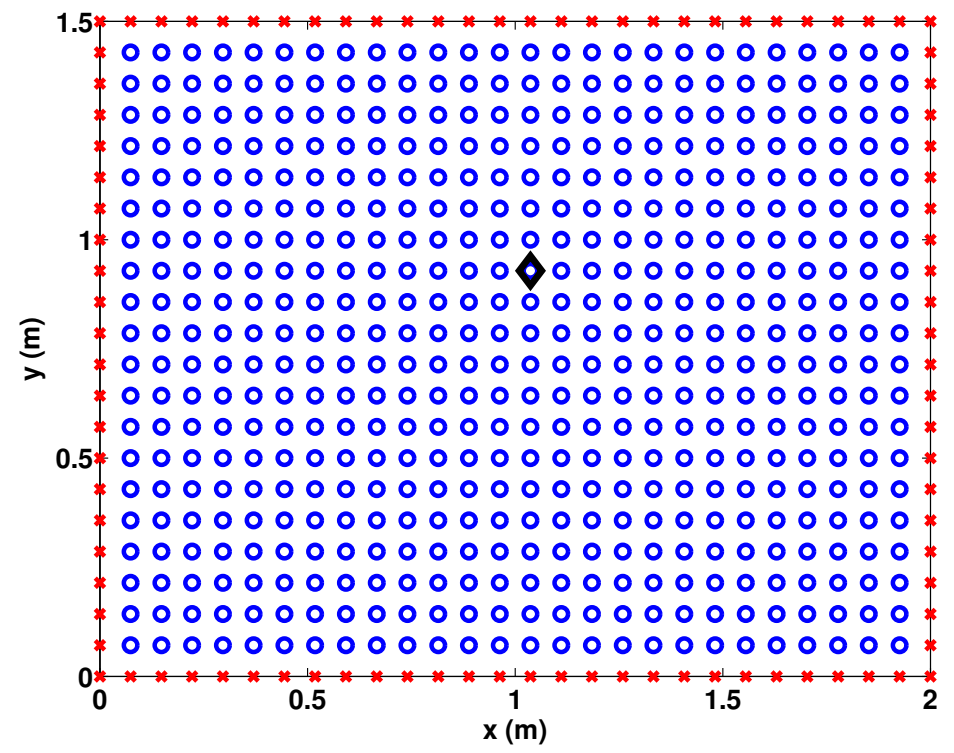

Figure 7: Definition of the selected zones - (o) zone 1 (sparse), $(\times)$ zone 2 (smooth) and $(\diamond)$ location of the point force

Following the considerations described in section 3.2.1, one sets $\left(q_{1}, q_{2}\right)=$ $(0.5,2)$. When choosing these values, one obtains the identified force vector presented in Fig. 8. It is noteworthy that the identified force vector is very similar to the reference one, meaning that chosen values are well adapted to describe the spatial distribution of sources in each selected zones. From a numerical standpoint, the immediate consequence of the accordance of the local priors with the actual local spatial distribution of sources is the decrease in the number of iteration of the GIRLS algorithm [8]. Indeed, only 11 it- 
erations have been required to reach the tolerance and the identified force amplitude $\widehat{\mathrm{F}}_{0}$ is equal to $0.995 \mathrm{~N}$ instead of $1 \mathrm{~N}$.

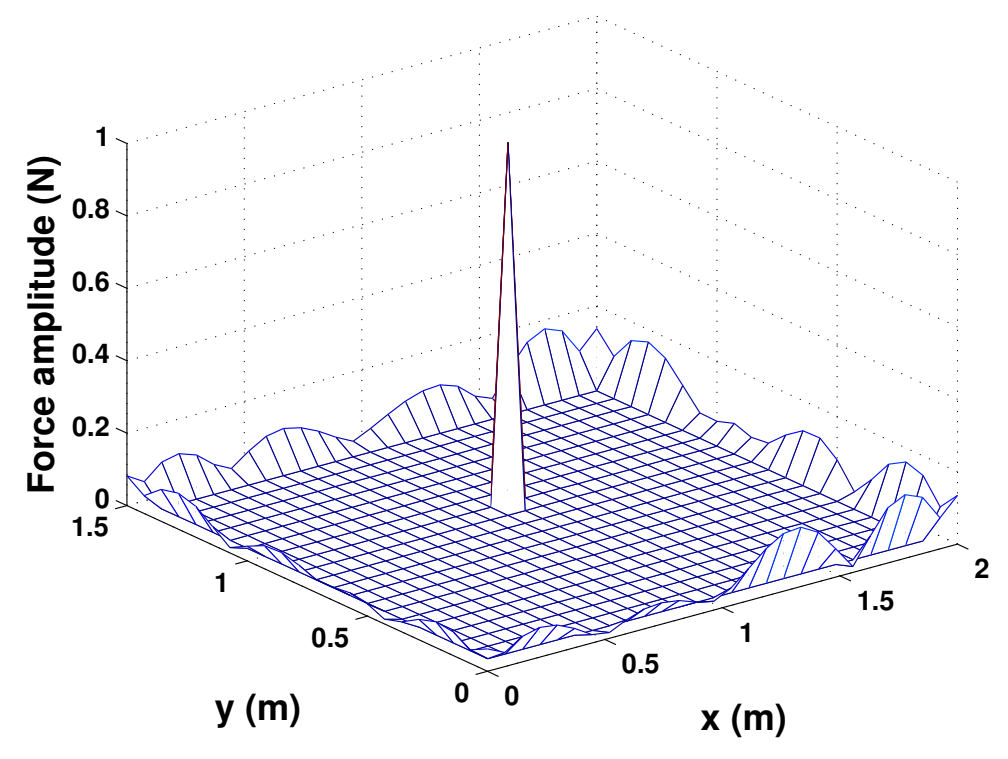

Figure 8: Identification of the force vector $\widehat{\mathbf{F}}$ at $450 \mathrm{~Hz}$ from corrupted data when using local priors on the spatial distribution of sources - $\left(\mathrm{q}_{1}, \mathrm{q}_{2}\right)=(0.5,2)$ and $\overline{\mathbf{L}}=\mathbf{I}$

This result can easily be explained by adopting the Bayesian paradigm. From this particular point of view, it can be noticed from Eq. (2) that the more prior information on the noise and the nature of the sources is meaningful, the more the confidence in the reconstruction is high. Numerically, this implies that the convergence of the GIRLS algorithm to a reliable solution is faster when choosing proper values of the shape parameters $q_{i}$.

The analysis presented above has been performed for a frequency that do 
not correspond to a natural frequency of the plate. However, it should be noted that source identification at natural frequencies of a lightly damped structure is generally far more difficult, since at these frequencies the shape of the vibration response of the structure is close to that of the corresponding mode shapes. Consequently, the nearfield information is somewhat masked. From the standpoint of the identification process, this can be regarded as an increase of the noise level. To assess the ability of the proposed approach to perform consistent identification at the natural frequencies of the structure, the identification procedure is applied on the test case described previously, but for resonances of the plate, namely $85 \mathrm{~Hz}, 278 \mathrm{~Hz}$ and $572 \mathrm{~Hz}$. The corresponding identifications are presented in Fig. 9. Obtained results show that for each natural frequency the shape of the identified force vectors are consistent with the reference ones. However, one has to noticed that the

amplitude of the identified point force $\widehat{F_{0}}$ is generally underestimated. Indeed, the identified amplitude is around $0.83 \mathrm{~N}$ instead of $1 \mathrm{~N}$ for the three cases. Nevertheless, these results show that the proposed method allows having credible information on the sources acting on the structure at resonance frequencies.

\section{Experimental validation}

The structure of interest is a steel parallelepiped box, excited on one of its faces by a shaker supplied by a white noise and equipped with a force sensor, as presented in Fig. 10. The main features of the proposed experimental validation are listed in table 3 .

In this experiment, the source identification is performed on the excited 


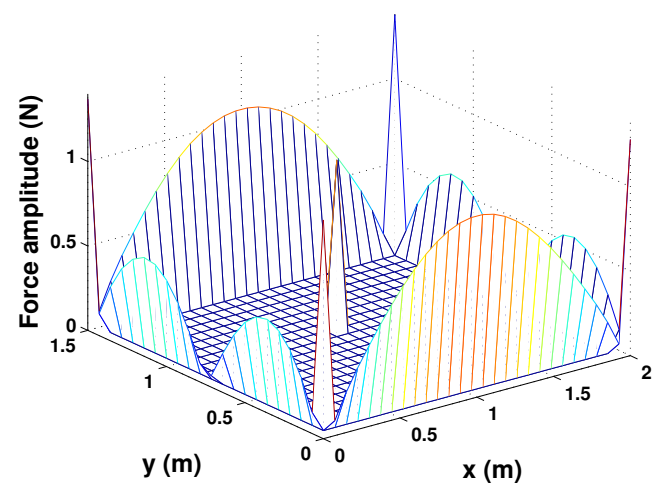

(a)

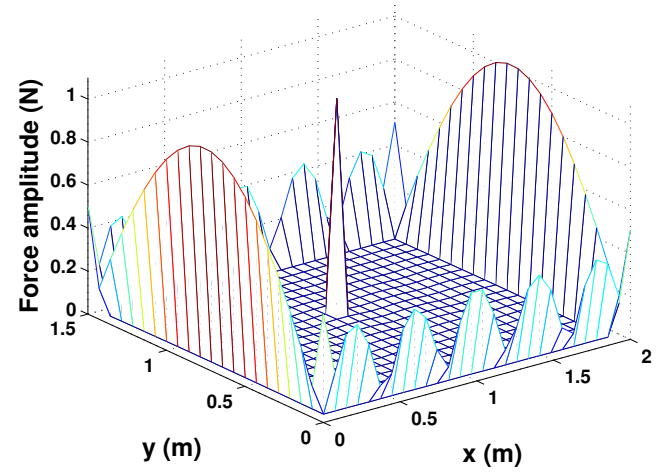

(c)

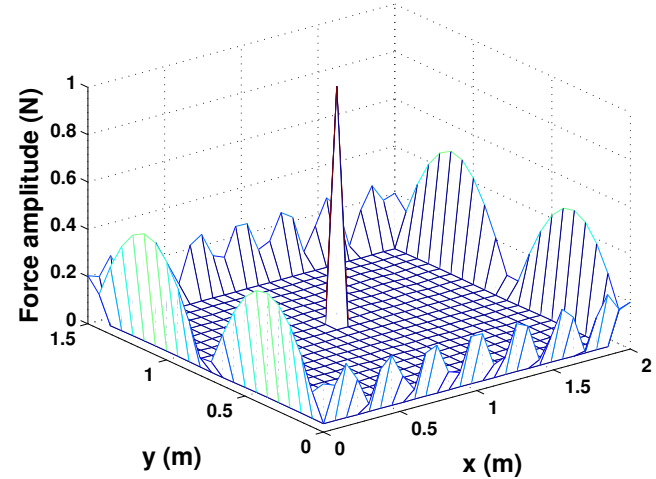

(e)

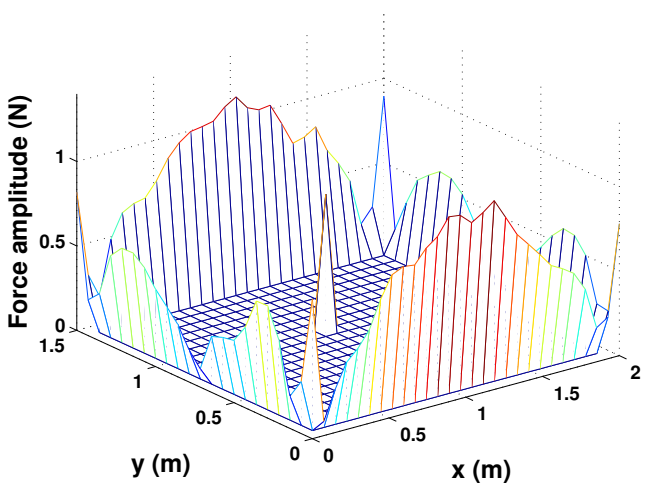

(b)

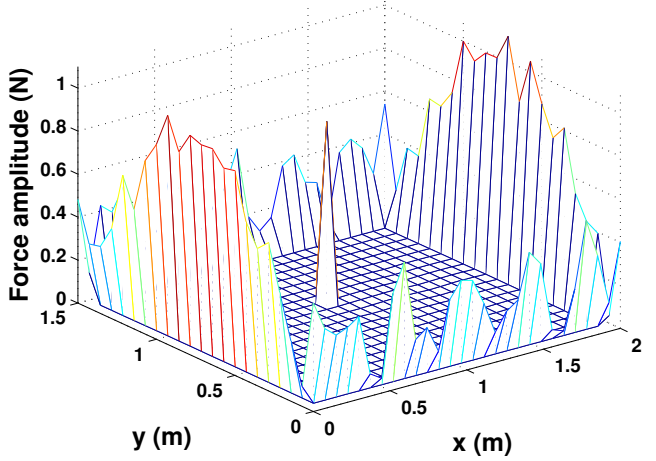

(d)

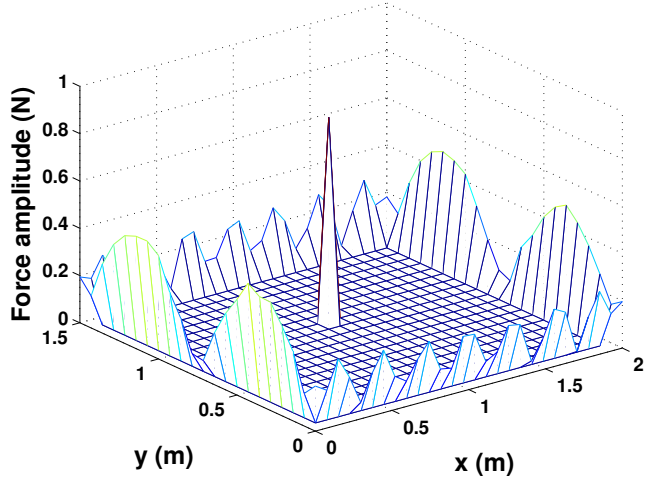

(f)

Figure 9: Identification of the force vector $\widehat{\mathbf{F}} 3$ at resonance frequencies of the structure - (a) Reference at $85 \mathrm{~Hz}$, (b) Identification at $85 \mathrm{~Hz}$, (c) Reference at $278 \mathrm{~Hz}$, (d) Identification at $278 \mathrm{~Hz}$, (e) Reference at $572 \mathrm{~Hz}$ and (f) Identification at $572 \mathrm{~Hz}-\left(\mathrm{q}_{1}, \mathrm{q}_{2}\right)=(0.5,2)$ and $\overline{\mathbf{L}}=\mathbf{I}$ 


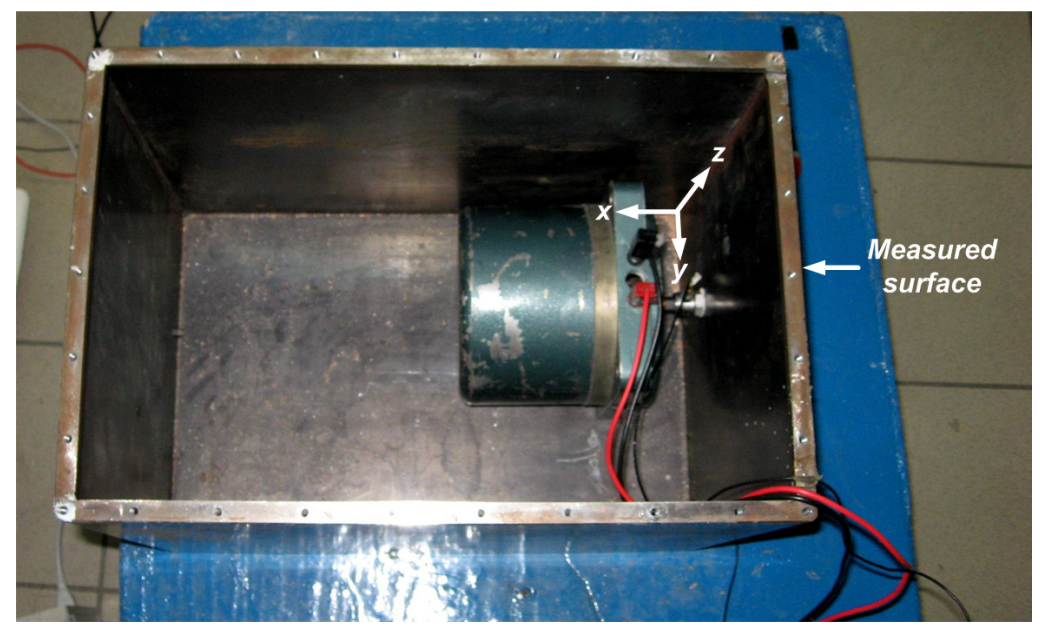

Figure 10: Experimental set-up

Table 3: Experiment parameters

\begin{tabular}{ll}
\hline Parameters & Values \\
\hline Length of the parallelepiped & $L_{x}=0.45 \mathrm{~m}$ \\
Width of the parallelepiped & $L_{y}=0.3 \mathrm{~m}$ \\
Height of the parallelepiped & $L_{z}=0.35 \mathrm{~m}$ \\
Wall thickness & $h=0.005 \mathrm{~m}$ \\
Young's modulus & $E=2.1 \times 10^{11} \mathrm{~Pa}$ \\
Density & $\rho=7800 \mathrm{~kg} \cdot \mathrm{m}^{-3}$ \\
Location of the force & $\left(y_{0}, z_{0}\right)=(0.10 \mathrm{~m}, 0.09 \mathrm{~m})$ \\
Studied frequency & $f_{0}=600 \mathrm{~Hz}$ \\
\hline
\end{tabular}


surface only [see Fig 10]. Measurements of the vibration field were carried out with a scanning laser vibrometer on a grid of $19 \times 22$ points along $y$ and $\mathrm{z}$ directions respectively using the signal as phase reference. In all the subsequent identifications, the measured vibration velocity field is normalized to the force signal delivered by the force sensor. By doing so, the amplitude of the identified point force $\widehat{\mathrm{F}}_{0}$ should be equal to 1 . Regarding the FE mesh used to model the dynamic behavior of the excited surface, it has been designed so as to perfectly match the measurement mesh. Hence, it is made up of 378 linear quadrilateral shell elements, making the model theoretically valid for frequencies below $5000 \mathrm{~Hz}$. The corresponding FE model with free boundary conditions has then been used to compute the transfer functions matrix $\mathbf{H}$, considering that transverse motions are the only available data.

As for the numerical validation, let us start by identifying the force vector $\widehat{\mathbf{F}}$ using a global prior on the nature of the sources to identify. In the present case, it can be noticed that the analysis of the experimental set-up suggests that the spatial distribution of sources acting on the studied part of the structure is similar to that of the numerical test case. Consequently, it is natural to choose $q=1.1$ and $\overline{\mathbf{L}}=\mathbf{I}$. After 85 iterations, one obtains the identified force vector presented in Fig. 11. It can be noticed that the spatial distribution of the point force is spread around its actual location, indicating that the global a priori is not perfectly adapted to identify localized sources. On the contrary, the spatial distribution of reaction forces is consistent with what is expected when analyzing the experimental set-up. It should however be noted that the identified forces at boundaries are the sum of pure reaction 
forces and equivalent forces due to the potential existence of boundary moments, because a force-only model of the structure is adopted here. As for the point force, one can, nevertheless, suspect that the identified amplitudes are underestimated, since the global a priori tries to reflect the compromise between the singularity of the point force and the smoothness of the reaction forces at boundaries.

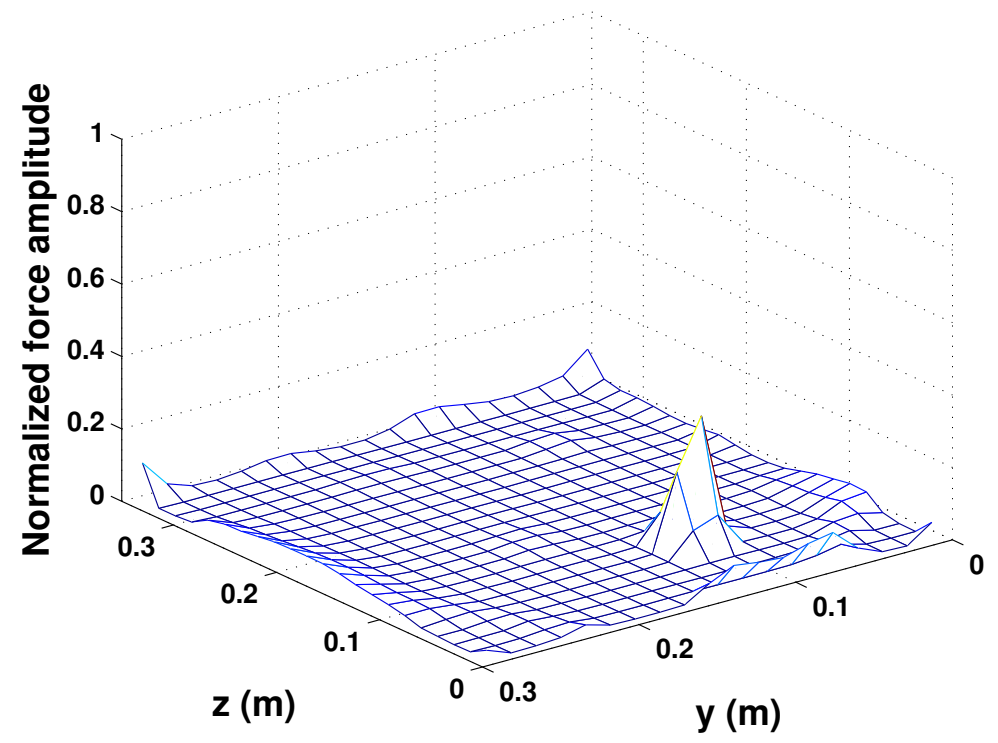

Figure 11: Experimental identification of the force vector $\widehat{\mathbf{F}}_{\mathbf{c}}$ at $600 \mathrm{~Hz}$ using a global prior on the spatial distribution of sources $-q=1.1$ and $\overline{\mathbf{L}}=\mathbf{I}$

As observed in the numerical validation, a better identification can be obtained by using properly our a priori on the nature and the location of sources acting on the structure. From a careful analysis of the experimental set-up, one can infer that the target force vector is very sparse except in the vicinity of the boundaries of the studied plate, where the spatial distribution 
of the reaction force is likely to be smooth. Accordingly, two identification regions can be distinguished as illustrated in Fig. 12. The selected regions have the same characteristics as those defined in the numerical validation. This observation allows assuming that $\left(q_{1}, q_{2}\right)=(0.5,2)$.

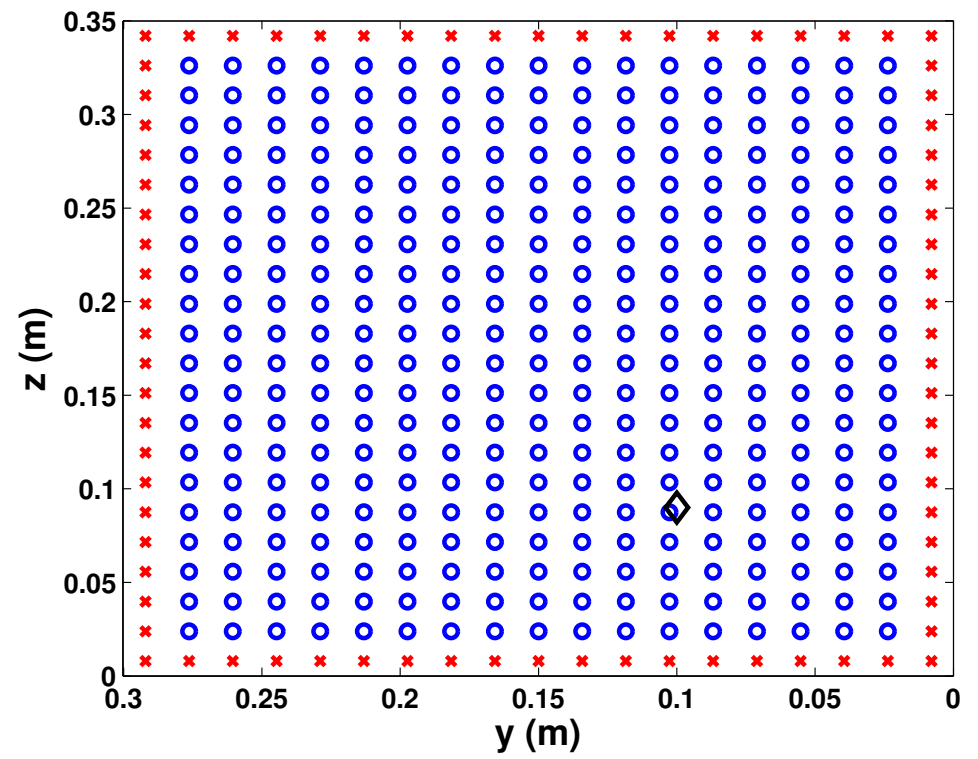

Figure 12: Definition of the selected zones - (o) zone 1 (sparse), $(\times)$ zone 2 (smooth) and $(\diamond)$ location of the point force

As shown in Fig. 13, the normalized amplitude and the location of the point force are well identified since $\widehat{\mathrm{F}}_{0}=0.93$ and $\left(\widehat{\mathrm{y}}_{0}, \widehat{\mathrm{z}}_{0}\right)=(0.1026 \mathrm{~m}, 0.0875 \mathrm{~m})$. Similarly, the reaction forces at boundaries remain consistent with our expectations. Finally, it can be noticed in passing that only 26 iterations have been necessary to reach the tolerance, confirming that properly exploiting the spatial information on the nature and the location of sources allows improving both the quality of the identification and the performance of the 
GIRLS algorithm.

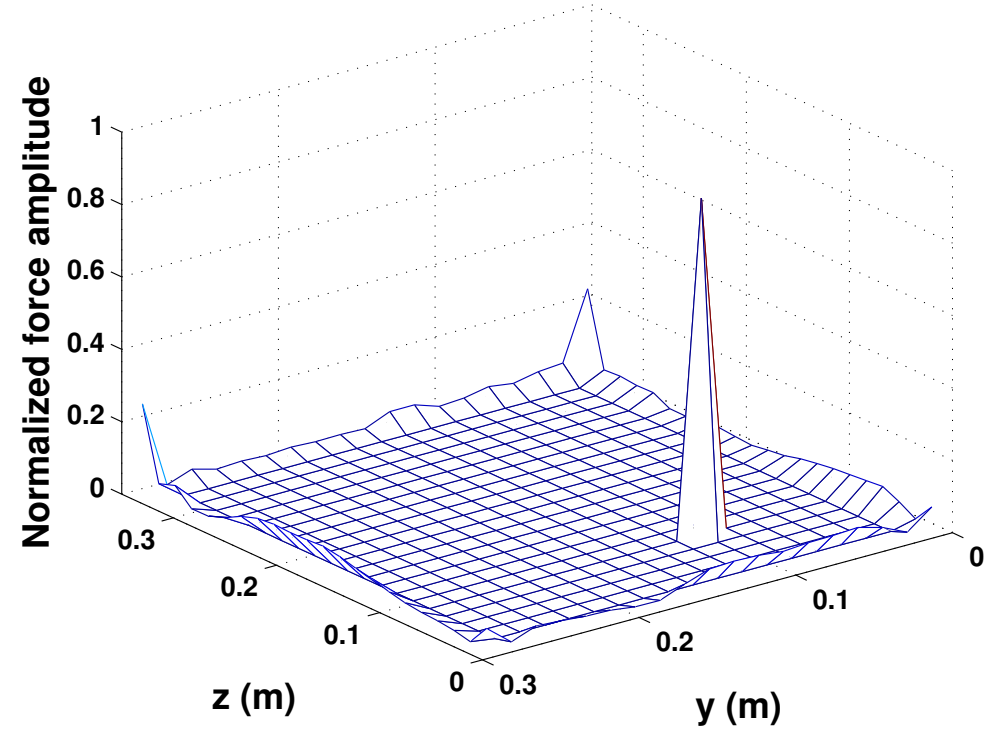

Figure 13: Experimental identification of the force vector $\widehat{\mathbf{F}}_{\mathbf{c}}$ at $600 \mathrm{~Hz}$ using local priors on the spatial distribution of sources - $\left(q_{1}, q_{2}\right)=(0.5,2)$ and $\overline{\mathbf{L}}=\mathbf{I}$

As for the numerical validation, the previous analysis has been performed outside natural frequencies of the structure. Nevertheless, this analysis is not sufficient to validate the proposed methodology in an experimental context, since identifying sources outside resonance frequencies is not the more challenging case. To have better insights on the identification ability of the approach, identifications are performed at resonance frequencies of the structure, namely $86.25 \mathrm{~Hz}, 212.5 \mathrm{~Hz}$ and $453.75 \mathrm{~Hz}$. The results presented in Fig. 14 clearly show that the corresponding identifications are qualitatively consistent with our expectations. Furthermore, it should be noted that the location of the point force is still well identified, while its normalized ampli- 
tude is underestimated since it is around 0.75 for the three cases presented here.

Finally, to give a comprehensive overview of the proposed identification strategy, the force spectra identified at the point force location is given in Figure 15. In an ideal situation, the identified force amplitude should be equal to 1 at each studied frequency. It is obviously not the case in the present validation. However, one can notice that the identified force spectra is reasonably close to the target value, as the discrepancy varies from $-5 \mathrm{~dB}$ to $2 \mathrm{~dB}$ over the entire frequency range and the frequency-averaged force amplitude is approximately equal to 1.02. Consequently, all these results are encouraging since credible results are obtained even with a model that does not strictly correspond to the actual structure.

\section{Conclusion}

In the present paper, a general methodology has been presented for identifying mechanical sources acting on a structure. The initial motivation of this study was to develop a method able to fully exploit the spatial information on the nature and the location of sources. Indeed, in the vast majority of the methods available in the literature, such an information remains unused, whereas it is generally roughly known in practical situations. To remedy this problem, we have used the Bayesian paradigm to derive a group generalized Tikhonov regularization, that allows introducing local a priori on the spatial

distribution of sources. In doing so, the identification procedure is expected to be more flexible and accurate, since the a priori can be precisely adapted 


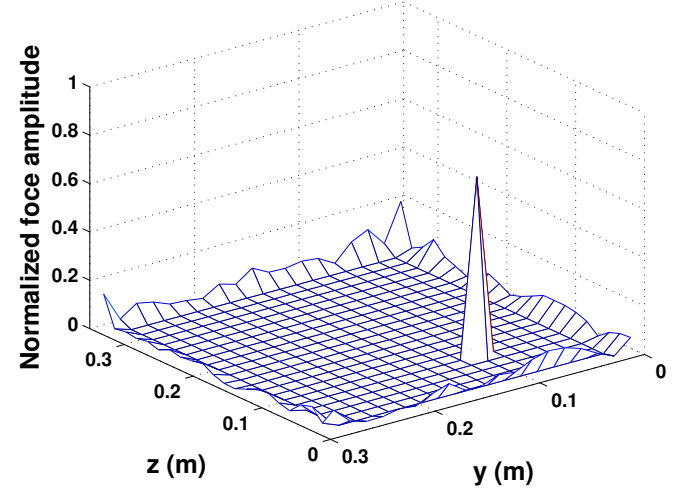

(a)

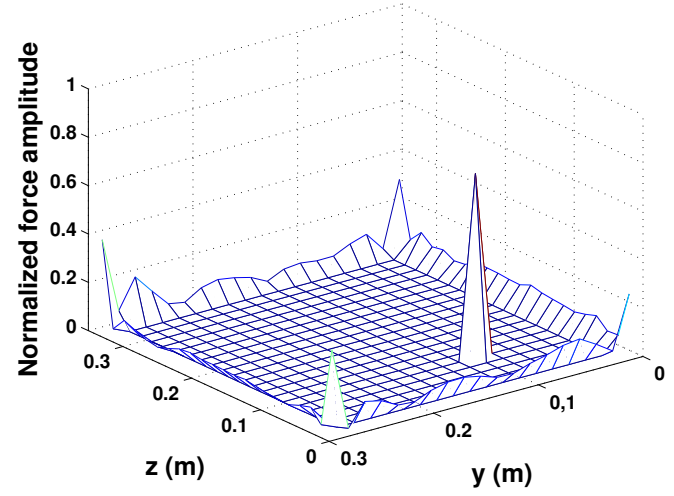

(b)

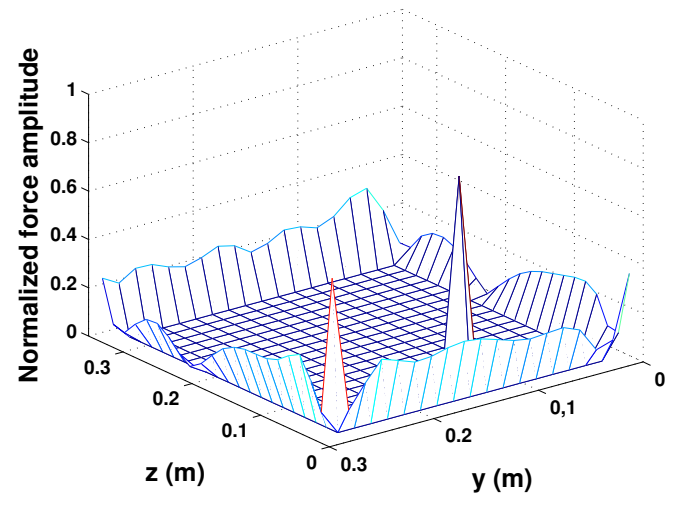

(c)

Figure 14: Experimental identification of the force vector $\widehat{\mathbf{F}}$ at resonance frequencies of the structure - (a) Identification at $86.25 \mathrm{~Hz}$, (b) Identification at $212.5 \mathrm{~Hz}$, (c) Identification at $453.75 \mathrm{~Hz}-\left(\mathrm{q}_{1}, \mathrm{q}_{2}\right)=(0.5,2)$ and $\overline{\mathbf{L}}=\mathbf{I}$ 


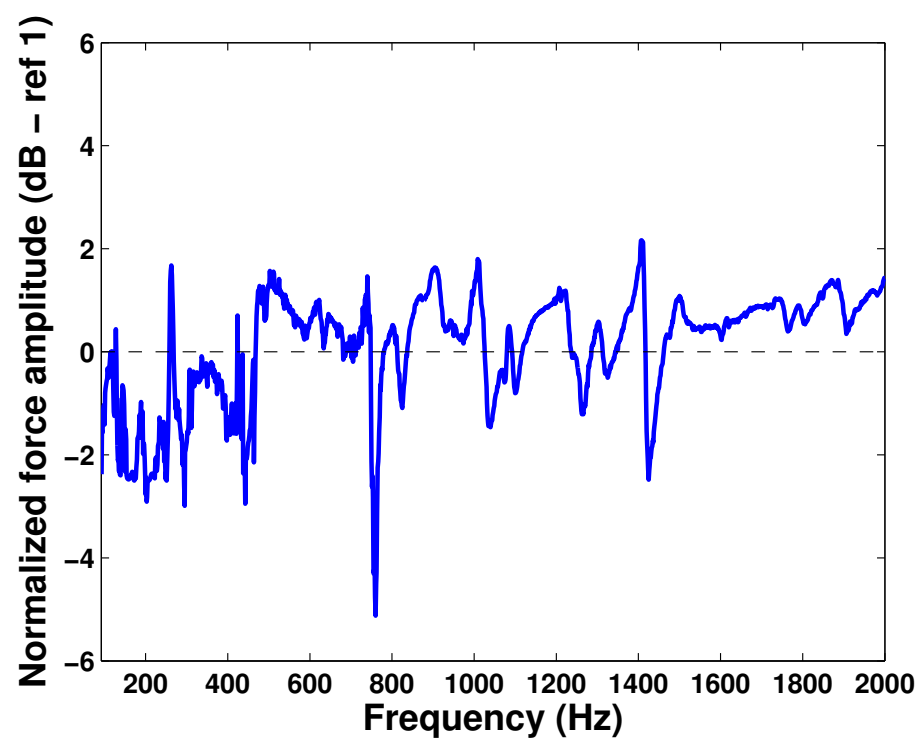

Figure 15: Force spectra identified at the point force location between $80 \mathrm{~Hz}$ and $2000 \mathrm{~Hz}$

to each zone of the studied structure. From a numerical standpoint, a Generalized Iteratively Reweighted Least-Squares (GIRLS) algorithm has been used to efficiently deal with both convex and non-convex problems, that can arise with such a formulation. Proposed numerical and experimental validations have highlighted the benefits of defining local priors instead of a global prior. In particular, it has been pointed out that properly exploiting the spatial information on the nature and the location of sources significantly improves the quality of identifications.

Two extensions of the identification strategy developed in this paper could be considered. First, the application of the proposed approach to non-linear structures by linearizing the problem around a certain state of the system and apply the GIRLS algorithm. Second, the implementation of a full Bayesian 
approach based on MCMC techniques for which the point estimates obtained with the proposed methodology could be considered as a valuable starting point.

\section{References}

[1] H. R. Busby and D. M. Trujillo. Optimal regularization of an inverse dynamics problem. Computers \& Structures, 63 (2):243-248, 1997.

[2] A. N. Thite and D. J. Thompson. The quantification of structure-borne transmission paths by inverse methods. Part 2 : Use of regularization techniques. Journal of Sound and Vibration, 264 (2):433-451, 2003.

[3] Y. Liu and W. Steve Shepard Jr. Reducing the impact of measurement errors when reconstructing dynamic forces. Journal of Vibration and Acoustics, 128:586-593, 2006.

[4] C. Renzi, C. Pezerat, and J.-L. Guyader. Vibratory source identification by using the finite element model of a subdomain of a flexural beam. Journal of Sound and Vibration, 332:545-562, 2013.

[5] C. Renzi. Identification Expérimentale de Sources vibratoires par Résolution du probleme Inverse modelisé par un opérateur Eléments Finis local (Vibratory sources identification by solving the inverse problem modeled by a local Finite Element operator). PhD Thesis, INSA de Lyon, 2011.

[6] C. Renzi, C. Pezerat, and J.-L. Guyader. Identification of vibration excitation using a regularized finite element operator and a deconvolution post-process. In Proceedings of Acoustics 2012, Nantes, France, 2012. 
[7] R. Molina, J. Mateos, and J. Abad. Prior models and the richardsonlucy restoration method. In The Restoration of HST Images and Spectra - II, 1993.

[8] M. Aucejo. Structural source identification using a generalized Tikhonov regularization. Journal of Sound and Vibration, 333(22):5693-5707, 2014.

[9] E. Zhang, J. Antoni, and P. Feissel. Bayesian force reconstruction with an uncertain model. Journal of Sound and Vibration, 331:798-814, 2012.

[10] P. Rodriguez and B. Wohlberg. An iteratively weighted norm algorithm for total variation regularization. In Proceedings of the 2006 Asilomar Conference on Signals, Systems and Computers, Pacific Grove, USA, 2006.

[11] I. Rish. An empirical study of the naive bayes classifier. In Proceedings of the IJCAI 2001 Workshop on empirical methods in artificial intelligence, Seattle, USA, 2001.

[12] A. Bjorck. Numerical methods for Least-Squares problems. SIAM, 1996.

[13] S. Boyd and L. Vandenberghe. Convex optimization. Cambridge University Press, 2004.

[14] R. Chartrand and V. Stavena. Nonconvex regularization for image segmentation. In Proccedings of International Conference on Image Processing, Computer Vision and Pattern Recognition (IPCV) 200\%, Las Vegas, USA, 2007. 
[15] M. Nikolova, M. K. Ng, S. Zhang, and W.-K. Ching. Efficient reconstruction of piecewise constant images using nonsmooth nonconvex minimization. SIAM Journal on Imaging Sciences, 1 (1):2-25, 2008.

[16] O. Scherzer. The use of Morozov's discrepancy principle for Tikhonov regularization for solving nonlinear ill-posed problems. Computing, 51:45-60, 1993.

[17] G. H. Golub, M. Heath, and G. Wahba. Generalized cross-validation as a method for choosing a good ridge parameter. Technometrics, 21 (2):215-223, 1979.

[18] J. Antoni. A Bayesian approach to sound source reconstruction: Optimal basis, regularization, and focusing. Journal of the Acoustical Society of America, 131 (4):2873-2890, 2012.

[19] P. C. Hansen. Rank-Deficient and Discrete Ill-Posed Problems: Numerical Aspects of Linear Inversion. SIAM, 1998.

[20] P. Rodriguez and B. Wohlberg. Efficient minimization method for a generalized total variation functional. IEEE Transactions on Image Processing, 18 (2):322-332, 2009.

[21] Q. Leclere. Acoustic imaging using under-determined inverseapproaches: Frequency limitations and optimal regularization. Journal of Sound and Vibration, 321:605-619, 2009.

[22] S.R. Ibrahim, A. Fregolent, and A. Sestieri. Structural force identification at unmeasured locations. In Proceedings of the 14th International Modal Analysis Conference, Dearborn, USA, 1996. 
[23] P. C. Hansen. Discrete Inverse Problems: Insight and Algorithms. SIAM, 2010. 